\title{
Corela
}

Cognition, représentation, langage

HS-19 | 2016

Le point de vue pris au mot

\section{Esprit du temps et coupe synchronique : la théorie du discours social}

\section{Marc Angenot}

\section{(2) OpenEdition}

\section{Journals}

\section{Édition électronique}

URL : http://journals.openedition.org/corela/4457

DOI : $10.4000 /$ corela.4457

ISSN : 1638-573X

\section{Éditeur}

Cercle linguistique du Centre et de I'Ouest - CerLICO

\section{Référence électronique}

Marc Angenot, «Esprit du temps et coupe synchronique : la théorie du discours social », Corela [En ligne], HS-19 | 2016, mis en ligne le 08 juin 2016, consulté le 19 avril 2019. URL : http:// journals.openedition.org/corela/4457 ; DOI : 10.4000/corela.4457

Ce document a été généré automatiquement le 19 avril 2019

\section{(c) (i) (2)(2)}

Corela - cognition, représentation, langage est mis à disposition selon les termes de la licence Creative Commons Attribution - Pas d'Utilisation Commerciale - Partage dans les Mêmes Conditions 4.0 International. 


\title{
Esprit du temps et coupe synchronique : la théorie du discours social
}

\author{
Marc Angenot
}

\section{L'esprit du temps}

1 L'histoire des idées englobe deux sortes de démarches, que je vois à la fois contraires et complémentaires, aussi légitimes l'une que l'autre : elle est aussi bien celle des évolutions et des devenirs - des diachronies - que celle de la description en coupe temporelle d'un état de choses, d'un état de la culture intellectuelle, l'étude de la coexistence d'«idées " mises en "discours», de "représentations » et de «croyances» en synchronie (ce ne saurait être ici le lieu de creuser cette polysémie confuse et floue qui depuis plus d'un siècle accompagne l'historiographie intellectuelle).

2 Il serait à propos de revenir sur la vieille notion de Zeitgeist, d'«Esprit du temps » ou «Esprit de l'époque », notion justement abandonnée dans son usage de jadis, organiciste et élitiste: les idées d'un temps appréhendées comme une sorte de "rencontre au sommet » dans une datcha transcendantale, loin des foules, entre de Grands penseurs. La notion, tout idéaliste qu'elle apparaît, serait néanmoins à retravailler en la rapprochant de nombreuses autres non moins floues, celles de «climat intellectuel », de «sensibilité d'époque » et autres formulations intuitives familières aux historiens. On parlera aussi de la « vision du monde » qui prévaut à une époque, la Weltanschauung collective ${ }^{1}$.

On ne rencontre au reste que de rares ouvrages consacrés à la reconstitution de l'«esprit " d'une époque à travers l'hégémonie de certaines idées et «images" dominantes (et non à travers ses mœurs et ses institutions ou plutôt en refoulant celles-ci à l'arrière-plan) : la vision du monde élisabéthaine a été reconstituée par Eustace Tillyard (1943), celle des colons de la Nouvelle Angleterre au 17e siècle par Perry Miller (1954 [1982]). 
4 Toute étude en coupe synchronique, je le précise au passage, n'est pas tenue à adopter une visée englobante. Michel Foucault (1969: 207) pressentant le malentendu (qui n'a pas manqué), souligne d'emblée que, dans Les mots et les choses, il n'a voulu que décrire une « région d'interpositivité » entre grammaire, économie et histoire naturelle au milieu du 18e siècle; aucunement dégager ou extrapoler, à travers cette convergence épistémologique sectorielle, un quelconque "Esprit de l'époque», - opération qui lui semblait fallacieuse et impossible à mener.

5 «Vision du monde » revêt occasionnellement une connotation démocratique si je puis dire: le plébéien comme le bourgeois et l'aristocrate entretiennent tous quelque chose comme leur propre vision du monde inséparable d'une « expérience de vie » qui est celle de leur classe et leur milieu, de leur Lebenswelt. L'« univers mental» d'un meunier italien $\mathrm{du} 16 \mathrm{e}$ siècle (poursuivi par l'Inquisition, ce qui procure sur sa culture paysanne une documentation unique), reconstruit par Carlo Ginzburg (1976) dans Le fromage et les vers forme la vision du monde d'une personnalité peu banale issue d'une classe exclue du monde lettré - « univers mental » sur la reconstitution duquel, à travers la littérature de colportage, ont également travaillé naguère les Robert Mandrou et les Geneviève Bollème.

Somme toute, l'idée de Zeitgeist, loin de permettre d'amalgamer en un ensemble factice les grands penseurs d'une "génération" donnée, semble s'appliquer au contraire à merveille à la culture de masse. Le modèle d'une étude du Zeitgeist hexagonal au $20^{\mathrm{e}}$ siècle serait ici Roland Barthes (1957) avec son deuxième livre, les Mythologies ${ }^{2}$. Barthes se montrait un analyste innovateur et subtil face à un phénomène nouveau, la culture médiatique; il reconstituait avec talent l'« esprit » des années 1950 avec la photo de l'acteur de chez Harcourt, la margarine Astra, le Guide bleu, la nouvelle Citroën DS, le Sénégalais de Paris-Match, l'épopée du Tour de France, la psychagogie publicitaire des saponides et détergents, l'iconographie de l'abbé Pierre et la propagande poujadiste, objets hétérogènes qui contribuaient par leur inter-lisibilité à construire une "mythologie" de l'après-guerre.

7 Toute étude synchronique du Zeitgeist se met en tout cas en quête de « dénominateurs communs", d'éléments de cohésion et de coïntelligibilité, en quête des «lieux communs " d'une doxa, lettrée ou populaire, des «lieux» communs aux idées et idéologies qui circulent et s'affrontent. Elle se met en quête de présupposés, de valeurs axiomatiques communes, de «cadres » qui s'imposeraient à toute pensée, de paradigmes élémentaires répandus, qui vont permettre de décrire une coexistence et en quelque sorte un enfermement dans un état du pensable et du dicible. Elle cherchera à montrer, en dépit de leur autonomie apparente et de la diversité de leurs objets, l'appartenance des idées, des systèmes d'idées à leur époque, à l'époque qui les a vu « naître » et prospérer.

Tel me semble le principe fondamental qui guide l'histoire des idées britannique : elle vise la reconstitution rigoureuse du contexte original, $d u$ "contexte d'époque»: pour comprendre une pensée, une doctrine du passé, il faut les (ré-)immerger en quelque sorte dans l'ensemble de ce qui se disait et se débattait à leur époque, dans le milieu dont ils émanent et parmi ceux qui disputaient du même problème - et dès lors de proche en proche dans le discours social global du temps considéré. Pour comprendre une croyance, il faut mettre en contexte les types et paramètres de croyables qui coexistaient vaille que vaille à une époque donnée ${ }^{3}$.

9 C'est bien encore ce qu'a posé jadis comme une évidence Michel Foucault (1966:274) à propos de la pensée de Karl Marx, - proposition reçue naguère avec une surprise 
interloquée par la jeunesse «de gauche», venant d'un penseur qu'elle admirait: le marxisme nage dans le $19^{\mathrm{e}}$ siècle « comme un poisson dans l'eau » - en d'autres termes, la pensée de Karl Marx est incapable de "respirer » hors de ces eaux-là. Foucault s'était borné à un geste de profanation élémentaire : restituer Marx et le ou les marxismes à leur historicité. Il a choqué les croyants de naguère avec ce propos peu discutable même s'il ne l'a pas approfondi: le penseur Marx nage, de conserve avec tous les penseurs de son temps, dans le $19^{\mathrm{e}}$ siècle des Grands récits historicistes, il «appartient » à ces temps disparus, évanouis, oblitérés, et seulement à eux.

\section{La théorie du discours social}

10 C'est dans la mesure où nos travaux à Pierre-Yves Raccah et à moi, quoique inspirés par des traditions disciplinaires différentes - lui venant de la linguistique, la sémantique, mais aussi de la logique, des mathématiques - se rejoignent néanmoins sur la vaste problématique de la communication et du savoir discursifs que je propose de résumer ici certaines des démarches que j'ai élaborées et certaines questions abordées en laissant au lecteur le soin de déceler des complémentarités et des divergences.

11 Il se fait que j'ai consacré à l'approche synchronique de la culture d'idées plusieurs de mes livres: je vais donc revenir sur la problématologie de ces travaux. Vers la fin des années 1980, je me suis mis en tête d'élaborer une théorie du discours social (Angenot 1986, 1989a, 1989b, 1989c, 1990). La critique du discours social allait appréhender et analyser en totalité la représentation discursive du monde telle qu'elle s'exprime dans un état de société, production qui présuppose le système complet des "intérêts" dont une société est chargée. Il s'agissait à mon sens de construire une problématique et une méthodologie susceptibles de rendre raison de la totalité de ce qui s'écrit, qui s'imprime et se diffuse à un moment donné dans un état de la société " moderne ». Je cherchais à considérer dans sa totalité l'immense rumeur de ce qui se dit et s'écrit à un moment donné en embrassant tous les secteurs, toutes les disciplines, tous les "champs» discursifs. Je voulais ainsi donner une consistance théorique à l'objet intuitif dont j'ai parlé au début de ce chapitre : la « culture » d'une époque, le Zeitgeist. Un Zeitgeist non pas conçu comme le phénomène d'une Cause expressive ni comme le fait d'une élite, d'une poignée de grands esprits, de grands penseurs qui «exprimeraient» leur temps. (Il est certain toutefois que ce que je définis plus bas comme l'« hégémonie discursive » produit, impose et légitime certaines pensées comme de " grandes pensées » et va faire de certains penseurs l'« incarnation de leur époque ».)

La notion de synchronie dont je pouvais me réclamer en cherchant à rendre raison d'un état du discours social est évidemment opposée à celle de la linguistique structurale. La synchronie saussurienne est une construction idéaltypique formant un système homéostatique d'unités fonctionnelles. La synchronie dont je parle forme une contemporanéité en temps réel. S'il existe en tout temps, comme j'en fais l'hypothèse, une certaine régulation "systémique" (avec des guillemets) du discours social, une « division du travail », une division réglée des champs et des genres discursifs avec leurs dicibles propres, l'étude synchronique fait aussi apparaître des points d'accrochage, des conflits, des formations idéologiques émergentes et d'autres récessives, de l'archaïque et du nouveau et, sur l'échelle des « distinctions », du doxique trivial et du paradoxe distingué, du novateur superficiel et des antinomies profondes, - fugacement de l'« inouï ».... 
13 L'idée de considérer en totalité ce que dit une société, de répertorier ses dicibles et ses scriptibles, ses «lieux communs» et ses «idées chics» est une idée vieille comme la modernité. Une bonne partie des "prédécesseurs» de l'analyse du discours social est ainsi formée par des gens de lettres. Tout au long des deux siècles modernes, de Gustave Flaubert à Léon Bloy, à Robert Musil, à Nathalie Sarraute, on voit revenir le recensement et l'interrogation accablée des "i idées reçues ", on voit l'artiste se consacrer à l'« Exégèse des lieux communs ». Qu'il s'agisse de Proust (Un Amour de Swann est un épisode que l'ont peut dater de la présidence de Jules Grévy vers 1887-88) ou de l'Homme sans qualité, ou encore des Fruits d'Or, de Vous les entendez, ce sont des romanciers qui ont avec le plus de subtilité écouté et transcrit la vaste rumeur hétérologique et cacophonique des langages sociaux.

14 L'élaboration de la théorie dont je parle a été appuyée sur un vaste « travail de terrain ». J'ai procédé à l'analyse systématique de la chose imprimée produite en langue française au cours d'une année que j'avais choisie avec quelques bonnes raisons contingentes, l'année mil huit cent quatre-vingt neuf. Mil huit cent quatre-vingt-neuf était simplement à mon sens une année prometteuse, une année-charnière de la triste «Belle époque »: c'est tout à la fois l'année du centenaire de la Révolution, l'année de l'Exposition universelle, de la Tour Eiffel, l'année de la résistible ascension du Brav' général Boulanger, l'année du « Drame de Meyerling » et de bien d'autres événements prégnants.

\section{L'hégémonie discursive}

15 À première vue, la vaste rumeur des discours sociaux donne l'impression du tohu-bohu et de la cacophonie, d'une extrême diversité de thèmes, d'opinions, de langages, de jargons et de styles. C'est à cette multiplicité, cette " hétéroglossie » ou " hétérologie » que la pensée de Mikhaïl M. Bakhtine s'est arrêtée. Au-delà de cette cacophonie apparente toutefois, ma démarche a consisté à rechercher des invariants et des récurrences, des présupposés collectifs, des "lieux communs ", - topoï koïnoï, c'est le mot d'Aristote dans les Topiques, - des dominantes, de l'homogène ou du moins du régulé, des principes de cohésion, des contraintes et des coalescences qui font que le discours social tel que je l'extrapole n'est pas une juxtaposition de formations discursives autonomes, mais apparait comme un espace d'interactions où des impositions de thèmes interdiscursifs omiprésents et de «manières de voir » et de dire viennent apporter au Zeitgeist une sorte d'unité « organique » en fixant les limites de l'argumentable, du narrable, - du pensable. Le discours social, dans sa diversité apparente, occupe et balise cet espace du diciblepensable. Nous pouvons lui appliquer une formule de Saint-Paul, In eo movemur et sumus, en lui nous évoluons et nous sommes; il est le médium obligé de toute pensée "publique », de toute expression même paradoxale.

16 Dans le "marché discursif» d'une époque donnée, il y a sans doute des objets thématiques et des formations discursives infiniment divers par leur statut social, leur régime cognitif, leurs destinataires. Il y a les lieux communs de la conversation, les grosses plaisanteries du Café du commerce, les chansons du café-concert, les espaces exotériques (eux-mêmes stratifiés en terme de degrés de "distinction ») du journalisme et des doxographes de "l'opinion publique » et de l'«actualité », du Gaulois lu par la duchesse de Guermantes au Petit journal de son concierge, aussi bien que les formes éthérées et prestigieuses, ou même tout à fait ésotériques et inaccessibles au commun, de la recherche esthétique, de la spéculation philosophique, de la formalisation scientifique. 
Il y a aussi bien les doctrines politiques et sociales établies qui s'affrontent en tonitruant que les murmures périphériques de groupuscules doctrinaires dissidents que les petites revues satiriques évoquent parfois avec un ébahissement goguenard. Tous ces lieux de discours sont pourvus en un moment donné d'acceptabilité et de "charmes », ils ont une efficace sociale et des publics captifs dont l'habitus acquis comporte une sensibilité à leurs influences, une capacité de les goûter et d'en renouveler le besoin.

L'objet que j'ai cherché à synthétiser n'était donc pas le tout empirique de surface, cacophonique et redondant. J'ai voulu extrapoler les règles de production et d'organisation des énoncés, les typologies et les topographies, relever les répertoires topiques et les présupposés cognitifs qui, pour une société donnée, organisent et délimitent le narrable et l'argumentable, si on pose que narrer et argumenter sont les deux modes prédominants du discours.

C'est ce qui fait que pour nous, avec l'intuitif « recul du temps », la psychopathologie de l'hystérie chez le Dr Charcot, la littérature boulevardière et libertine de Catulle Mendès, l'esprit d'Henri Rochefort et celui d'Aurélien Scholl, les romans d'Émile Zola et ceux de Paul Bourget, les factums antisémites d'Édouard Drumont et les chansons du café-concert de Paulus nous semblent, tant par leur forme que par leur contenu, appartenir à la même époque (alors que nous serions en peine de dire ce qui précisément les rapproche) - cette époque que les contemporains avaient appelée, avec un sentiment d'angoisse crépusculaire, la «Fin de siècle » et qu'une génération plus tard, après la guerre, on identifiera avec nostalgie comme «La Belle Époque », le début de cette Belle Époque qui va de la présidence de Carnot à celle de Félix Faure.

Quand je parle de constantes et de régulations, je m'empresse d'ajouter qu'un état de culture n'est pas à mes yeux un "système", mais une homéostase relative avec des fluctuations et des conflits. Il dégage néanmoins une «atmosphère » engendrée par une vaste agglomération de croyances plus ou moins co-intelligibles, de topoï, présupposés, idées reçues, stéréotypes, valeurs, mythes, le tout régulé par des présupposés fondamentaux. La cohérence relative de tout ceci découle d'un bricolage régi par le « sens commun » prévalant à l'époque. Le discours social forme, sinon un système du moins un " marché » soumis à des tendances générales, un marché dont les secteurs en interaction sont perméables les uns aux autres et dont les régimes locaux de production du sens s'expliquent en partie par cette interaction et topographie mêmes. Renonçant à l'analyse sectorielle isolée de genres et de champs discursifs déterminés, - littéraire, philosophique, scientifiques, politique, journalistique, - mon analyse prétendait décloisonner la recherche, intégrer les "secteurs" discursifs particuliers dans un réseau interdiscursif global, prendre à bras le corps l'énorme masse cacophonique des langages qui viennent à l'oreille de l'homme-en-société et reconquérir ainsi une perspective totalisatrice. Le chercheur allait pouvoir identifier des récurrences et des dominantes, des manières de connaître et de signifier le connu qui sont le propre de cet état de société et qui transcendent la division des discours et faire voir comment, par exemple, certains idéologèmes prédominants en un moment donné reçoivent leur acceptabilité d'une grande capacité de mutation et de relance passant de la presse d'actualité au roman, au discours médical ou scientifique, à l'essai de philosophie sociale etc.

Le discours social a le monopole de la représentation de la réalité, cette représentation de la réalité qui contribue largement à faire la réalité ... et l'histoire. C'est justement parce que c'est affaire de monopole que le discours social d'une époque semble adéquat comme reflet du réel puisque « tout le monde » voit le réel et le moment historique à travers lui 
et, plus ou moins, de la même façon. La fonction des discours sociaux, fonction concomitante à leur monopole de représentation, est de produire et de fixer des légitimités, des validités, des publicités (de rendre publics des goûts, des opinions, des informations, des valeurs). Complémentairement, elle est de refouler et de censurer l'impensable. À la fonction "monopoliste" du discours social se subordonnent des fonctions dérivées de routinisation de la nouveauté, de convivialité nationale, d'identification distinctive des groupes et des milieux, de leurs goûts et de leurs intérêts.

Ce qu'on appelle une «culture » est composé de mots de passe et de sujets de mise, de thèmes dont il y a lieu de disserter, sur lesquels il faut s'informer et qui s'offrent non seulement aux "médias " mais à la littérature et aux sciences comme dignes de méditation et d'examen. L'hégémonie se présente ici comme une thématique, avec des savoirs vulgaires et des savoirs d'apparat, des "problèmes " préconstruits, des intérêts attachés à des objets dont l'existence et la consistance ne semblent pas faire de doute puisque tout le monde en parle.

Il n'est pas besoin d'épouser les mêmes idées pour respirer le même air. Il suffit qu'on s'accorde à tenir ceci ou bien cela pour réel : ce qui est digne d'être débattu. Par ce choix préalable aussi spontané qu'inconscient s'opère l'essentiel, qui est le partage entre le décisif et l'accessoire. (Debray $2000: 82$ )

Tout débat dans l'opinion "publique » ou en un secteur donné, si âpres que soient les désaccords, suppose un accord préalable sur le fait que le sujet " existe », qu'il «mérite " d'être débattu, - et qu'un commun dénominateur minimal sert d'assise aux dissensions et polémiques. On touche ici à ce qui est le plus perceptible dans une conjoncture, à ce qui étonne ou agace le plus le lecteur d'un autre pays ou d'une autre époque : de tous ces « objets » que l'on nommait, que l'on valorisait, que l'on décrivait et commentait combien n'apparaissent plus comme étant des réalités connaissables mais, avec le recul du temps, sont réduits au statut de « bibelots d'inanités sonores ».

23 À celui qui est immergé dans les discours de son époque, les arbres cachent la forêt. À assister aux débats acharnés en politique, aux confrontations d'esthétiques antipathiques l'une à l'autre, à percevoir les spécialisations et les spécificités, les talents et les opinions, la pression de l'hégémonie reste cachée. Ce qui est caché est le « système » sous-jacent et il faut que ce système soit tu pour que les discours possèdent leurs charmes et leur crédibilité.

L'hégémonie ce n'est aucunement la dominance quantitative, laquelle rendrait plus « audibles » à la fin du $19^{\mathrm{e}}$ siècle les poncifs du café-concert et la grosse blague des revues populaires que les subtils débats de la Revue des Deux Mondes. L'hégémonie est un ensemble de mécanismes unificateurs et régulateurs qui assurent à la fois la division du travail discursif et ses hiérarchies, et un degré d'homogénéisation des rhétoriques, des topiques et de la doxa transdiscursives. Ces mécanismes imposent sur ce qui se dit et s'écrit de l'acceptabilité et stratifient des degrés et des marques de légitimation et de distinction. L'hégémonie se compose donc des règles canoniques des genres et des discours (y compris leur marge des variances et déviances acceptables), des préséances et des statuts des différents discours eux-mêmes, des normes du bon langage (y compris, encore, le contrôle des degrés de distinction langagière, du haut style littéraire au toutvenant de l'écriture journalistique « concierge » comme disaient les lettrés), des formes acceptables de la narration, de l'argumentation et plus généralement de la cognition discursive et du répertoire des thèmes qui s'« imposent » à tous, mais de telle sorte que 
leur traitement ouvre le champ de débats et de dissensions eux-mêmes régulés par des conventions de forme et de contenu.

Les différents genres établis du discours social parlent à un destinataire implicite, lui aussi légitimé, et il n'est de meilleur moyen de le légitimer que de lui donner droit de regard sur ceux qui n'ont pas droit à la parole, « sur le dos » desquels cela parle : les fous, les criminels, les enfants, les femmes, les plèbes paysannes et urbaines, les sauvages et autres primitifs...

L'effet de « masse synchronique » du discours social surdétermine la lisibilité (le mode de lecture et d'interprétation) des textes particuliers qui forment cette masse. L'interlisibilité assure une entropie herméneutique qui fait lire les textes d'un temps (et ceux de la mémoire culturelle) avec une certaine étroitesse interprétative; celle-ci scotomise la nature hétérologique de certains écrits, elle aveugle à l'inattendu et tend à réduire le nouveau au prévisible. C'est en quoi les «idées nouvelles » risquent de passer inaperçues parce qu'elles sont abordées dans des cadres préconstruits qui offusquent ce qui se prêterait ici et là à une lecture différente.

La chose imprimée même est un instrument de légitimation en soi en un temps où les gens simples croient sans réserve à ce qui est « écrit sur le journal». Le discours social, dans l'unité et la cohérence relatives de son hégémonie, renferme un principe de communion, de convivialité. Il re-présente la société comme ensemble intelligible (en dépit de menaces angoissantes repérées par les "observateurs») et comme convivium doxique, et même les affrontements polémiques, savants ou triviaux, même les dissensions y contribuent. Le discours social produit une certaine interprétation commune de la conjoncture, il lui confère un sens dont débat le chœur des doxographes. Le discours social est toujours là, comme médiation, interposition du collectif préconstruit dans les rapports entre les humains. C'est ce que Flaubert a voulu faire sentir en narrant la première rencontre d'Emma Bovary et de Léon à l'Auberge d'Yonvillel'Abbaye : l'immersion totale des sentiments, des sensations, des désirs «individuels » dans l'aliénation de la doxa, du cliché romantique et des idées reçues.

\section{Topographie : Champs discursifs et division du travail}

Le discours social est divisé en champs et ces champs eux-mêmes forment un "système " de genres, de répertoires thématiques et de "styles", le tout formant ce que je désigne comme une topographie. Chacune de ces entités discursives peut se caractériser par une axiomatique propre, un champ (où elle s'insère et trouve ses antagonistes éventuels), un statut culturel, des attaches institutionnelles, une fonction dans la circulation des idéologèmes comme dispositif intertextuel spécifique, une idéologie immanente qui lui confère mandat et légitimation, une tradition propre, des intervenants attitrés avec leurs prestiges particuliers et leurs habiletés. L'unité d'un champ donné est celle d'un marché discursif autonome, c'est-à-dire un espace d'échange, de concurrences et de migrations d'énoncés, un espace de contiguïtés attestées par les affrontements mêmes qui s'y expriment, par des concurrences reconnues dans l'hostilité par les agents, collectifs et individuels, qui y opèrent. Chaque champ discursif dispose d'enjeux communs et constitue une communauté conflictuelle qui exploite des thèmes et des stratégies, exige des droits d'entrée, procure une homologation des produits et maintient un certain 
cloisonnement destiné à préserver l'identité canonique de la production. Chacun confère un statut aux agents qui y opèrent, lesquels forment une sorte de compagnonnage conflictuel autour d'enjeux communs. Les champs ne sont pas en effet des collectivités harmonieuses, mais des espaces concurrentiels où des idées "se posent en s'opposant ", où des pratiques se heurtent à d'autres pratiques, des thèses à des thèses antagonistes. $\mathrm{Vu}$ de l'extérieur, tout champ est un dispositif de raréfaction et de censure : il fixe ce qui y est nommable et inacceptable, approprié et inapproprié, "dans les formes" et inconforme. C'est à ce niveau que se détermine aussi ce qui pourra passer des « influences étrangères » et comment celles-ci (darwinisme, wagnérisme, roman russe, psychologie allemande de l'inconscient ${ }^{4}$ théâtre ibsenien...) seront filtrées, nationalisées.

La logique de ces systèmes est totalement historique: rien n'est par nature d'ordre " littéraire " plutôt que d'un autre ordre. C'est l'arbitraire culturel de 1889 qui fait que "le Salon », la critique d'art est un genre littéraire de plein droit, de même qu'avec Édouard Drumont, le pamphlet antisémite appartient bel et bien marginalement au système littéraire. Un champ est un marché symbolique où des pratiques vont s'échanger et des capitaux s'accumuler selon une fixation immanente des "prix». Ces espaces structurés, avec leurs agents propres, où se déplace de l'énergie sociale et où agissent des forces d'un genre déterminé, se déploient autour d'appareils et d'institutions donnés.

Il convient de transposer une notion économique classique en parlant de division du travail discursif. Rien de ce qui se dit et s'écrit dans une société ne peut se dire identiquement partout. Ce qu'écrit un philosophe ou un essayiste, il ne pourrait le «traduire» en vaudeville ou en élégie ! La division du travail discursif impose en principe un interdit qui est celui «du mélange des genres »: ne pas politiser la littérature, ne pas faire de la politique une mystique, ne pas poétiser le discours philosophique; à chacun son métier. Cependant, de multiples transgressions de cette règle peuvent s'observer. Sans que les secteurs discursifs soient des vases communicants, entre eux s'établiront des " capillarités ", des vecteurs de perméabilité ; des « chicanes » contrôlent divers points de passage.

31 Pour prolonger l'image territoriale, on peut noter en certains lieux des points d'accrochage, parcourir des zones floues qui sont plus ou moins res nullius ou en condominium, par exemple vers 1889 certains « terrains » situés entre la littérature et le journalisme (du côté de la chronique, de la satire, de la critique d'art) et assister à de véritables conflits inter-sectoriels. On signalera par exemple l'âpre dispute entre philosophie (possédante) et médecine (prétendante) pour s'annexer le secteur de bon rapport de la psychologie, en voie d'être modernisée en « psychologie expérimentale ».

Les clivages mêmes qu'une société reçoit comme plus ou moins évidents et immuables ont une histoire qui les relativise. À commencer par le paradigme topographique le plus fameux et de plus longue durée de la France : droite / gauche. En termes de topographie politique, l'idée (que perpétuent bien des travaux acadéliques) d'un affrontement séculaire entre des idées de droite et de gauche par essence, séparées par une opposition nette depuis les Lumières et les Anti-lumières, est un de ces mythes manichéens que toute analyse contredit totalement. Ce qui était classé progressiste à un moment donné devient réactionnaire et vice-versa. Le retour à la terre, fantasme hautement "réac" du temps du Régime de Vichy, est valorisé et reformulé comme progressiste, des soixante-huitards aux écolos... Contre toute essentialisation intemporelle, il faut faire l'histoire des avatars, des glissements-recyclages de certaines idées entre droite et gauche, et même extrême droite et extrême gauche en moyenne durée. 


\section{Interdiscursivité}

thématiser c'est mettre en connexion un objet doxique avec d'autres déjà-là, déjà parlés, jugés, évalués. C'est ce que j'ai essayé de montrer dans Angenot (1986) : on ne peut parler du sexe en 1889 , - des « aberrations sexuelles », des "vénalités ", des " assouvissements " et des « ruts » pour évoquer le langage d'époque - qu'en le faisant travailler sur d'autres idéologèmes qui sont alors actifs : la décadence des mœurs, la lascivité juive, les monstres en soutane, l'imperfectibilité de la race noire, les stupres paysans, l'anonymat urbain, les à-vau-l'eau sociaux... Dès lors, toute formation discursive appelle en synchronie une herméneutique interdiscursive. L'hystérie (selon l'École de la Sapétrière) parle, dès qu'on en pousse l'exégèse, d'autre chose que d'un désordre neuropathologique, de même que les discours de la polissonnerie boulevardière parlent d'autres choses aussi que d'Éden prostitutionnel et de chronique du demi-monde. «Une» idéologie ne se renferme jamais sur elle-même; tout se tient, tout se connecte et les configurations de sociogrammes suggèrent des parcours, invitent à explorer des secteurs doxiques contigus, toute analyse exige la maîtrise subliminale du système thématique global que forme le discours social.

On introduira ici le concept de migrations pour décrire la diffusion de certaines «idées ", « valeurs » et procédés rhétoriques d'un genre discursif à l'autre, d'un champ à un autre, avec l'adaptation de ces entités migrantes à la logique du champ d'arrivée et à son héritage de formes propres. Ainsi, du «médical» en 1889 se trouve absorbé, intégré et adapté aux thématiques littéraires; des formes littéraires, romanesques notamment se trouvent empruntées par le journalisme ou par l'écriture scientifique; des sujets d'actualité en migrant du journalisme à des secteurs plus "ésotériques ", subissent un avatar philosophique ou artistique ou savant. Tout genre du discours est alors un dispositif interdiscursif, perméable à la migration d'éléments venus de discours contigus qu'il adapte à son telos propre et partageant des stratégies avec des discours parents. La définition interne d'un genre du discours doit être complétée par une approche de celuici en tant que dispositif intertextuel, en contiguïté avec d'autres formations discursives dont il sélectionne, absorbe, intègre et altère certains idéologèmes, certains micro- récits et certains schémas persuasifs.

Petit exemple : «Struggle for Life» - En 1889, l'idéologème de « la lutte pour la vie » est un axiome dans les sciences naturelles (entendu de façons différentes par les diverses écoles post-lamarckiennes qui peinent à comprendre l'épistémè non-téléologique de Charles Darwin); il procure un micro-récit largement exploité par les genres romanesques et dramatiques ; il est devenu un moyen d'exégèse de l'« actualité » pour le journaliste. Il a sa variante libérale et sa variante socialisante. Il est polyvalent, versatile, sous l'apparence de l'identité, mais il impose aussi une certaine logique. Il n'est pas dépourvu toutefois de contenu minimal et possède des "atomes crochus", une virtualité de se connecter avec tel et tel autre idéologème disponible. Dès lors, parce qu'il n'est ni un simple instrument qui permettrait de 'penser ce qu'on veut', ni une monade à libre combinaison, il incline celui qui en use à certaines connexions, à certaines mises en relation, il a dans une conjoncture donnée, une valence qui prédétermine en partie l'usage qu'on peut en faire. Joubert comparait les pensées à des monnaies qui circulent dans la société, passant de cerveau en cerveau. La valeur d'échange des images, des idées et des opinions prime, dans le discours social, sur leur valeur d'usage. 

discursifs, plusieurs styles de thématisation convergent dans leur efficace et ne produisent que par leur coopération une évidence hégémonique. Prenons le cas, facile à esquisser en quelques lignes, de la misogynie façon «Belle époque»: des galanteries mufles du libertinage boulevardier aux diatribes positivistes de la médicalisation de la femme, aux avertissements des gens graves sur l'absurdité d'une émancipation contre nature, à la " production » des petites filles par les manuels scolaires, un grand nombre de dispositifs hétérogènes engendrent un noeud gordien d'idéologèmes intriqués - en stricte logique contradictoires parfois - mais qui sont coopérants et produisent ce que nous nommerons un sociogramme global. Le discours social ne tend pas vers de l'homogène, mais produit des hétérogénéités réglées et efficaces où diverses positions trouvent à contribuer.

\section{Sociogrammes}

ude Duchet $(1974,1979 a, 1979 b, 1980)$ le fondateur français de la sociocritique des textes, définit ce qu'il entend par sociogramme ${ }^{5}$ dans les termes suivants : «Ensemble flou, instable, conflictuel, de représentations partielles, centrées autour d'un noyau thématique, en interaction les unes avec les autres ». Duchet voit bien que l'objet essentiel qui s'offre à l'analyste des discours n'est pas d'abord ces grandes constructions doctrinaires auxquelles on a attribué le nom d'idéologies, pas plus que l'unité opératoire ne peut être ramenée d'emblée à des propositions élémentaires - maximes, idéologèmes, slogans, images - qui s'isoleraient de façon autonome dans l'univers des représentations discursives. L'objet premier pour l'analyste, ce sont ces convergences interdiscursives par lesquelles, dans l'hétérogénéité, dans l'antagonisme latent sinon la cacophonie, les différents discours et les différentes idéologies thématisent, figurent et interprètent simultanément certains aspects de la vie sociale. D'où ce modèle du sociogramme où, à partir d'un noyau thématique, divergent des vecteurs de représentations-interprétations portés par la logique des différents discours, le tout formant un ensemble instable, ne cessant de se transformer par dynamique interne et en phagocytant des éléments empruntés à des thématisations contiguës; ensemble dont les éléments sont porteurs d'enjeux et de débats; ensemble dont enfin les limites sont floues, dont les "frontières " ne sont aucunement étanches.

Illustration: Le sociogramme de la prostituée. - j'ai notamment étudié l'ensemble des mises en discours qui, en 1889, dans les lettres et dans les discours des publicistes et dans les disciplines savantes, opèrent sur «la prostituée» (Angenot 1986). On a noté bien souvent l'omniprésence de l'«amour vénal " comme thème romanesque et poétique, omniprésence qui va s'exacerbant dans le dernier tiers du $19^{\mathrm{e}}$ siècle. Reprenons synthétiquement la description des vecteurs porteurs de représentations de la prostitution. On signalerait d'abord, hors du domaine de la chose imprimée, le secteur de l'oralité masculine, de la " conversation de fumoir ", drainant des mythes, des anecdotes et des impensés avec tout l'investissement interactionnel de savoirs semi-clandestins, cyniques et gratifiants. Dans les discours d'appareil, en position de pouvoir et de légitimité savante, il y a le discours qui assume désormais la médicalisation et de la déviance criminelle et du sexe et, au point de convergence, celle du sexe prostitué.

Le discours médical se prolonge dans celui de tous les surveillants de l'ordre dont le bras séculier est la police des mœurs. Les débats entre réglementaristes et anti-

Corela, HS-19 | 2016 
réglementaristes (soucieux de ne pas abaisser le pouvoir d'état dans une sorte de proxénétisme honteux) s'affrontent sur ce vecteur et l'articulent à divers principes éthiques et politologiques. Le criminologue, promoteur d'une « science » en émergence, impose avec Cesare Lombroso le concept de "prostituée-née ", sorte de "bête humaine " atavique en régrès dans le progrès culturel et moral. La criminologie, construite sur l'analogie criminel / sauvage et criminel / enfant pervers, conçoit, à partir de là, la courtisane comme femme-enfant et femelle primitive, retrouvant selon sa logique propre deux topoï éculés de la fascination « littéraire » qu'exerce l'éternel féminin. Le paradigme de Lombroso est toutefois contrebattu par d'autres argumentations étiologiques qui remontent au début du siècle industriel et qui mobilisent des savoirs hétérogènes, sur l'ordre sexuel, la femme éternelle, les nécessités de la vie sociale, la nature des identités et des sensibilités de classe. Sur un tout autre vecteur, moins assuré de ses prérogatives vertueuses, mais tout aussi dynamique et éminemment producteur de représentations, il y a la vaste presse « boulevardière » où la philosophie de la parisianité chante l'apothéose de la cocotte, de l'horizontale, de la vie de noce et du libertinage distingué. Toute proche, on trouve la littérature « bien écrite » du lyrisme aphrodisiaque faisandé. La prostitution est construite aussi selon une logique du sensationnalisme dans le journalisme d'information où le thème de fait-divers de la fille éventrée, de l'Affaire Pranzini à Jack l'Éventreur, mêle en une suprême "expiation" la libido et le sang érotisé. Sur les périphéries, le contre-discours socialiste et anarchiste construit l'image d'une double exploitation par le hideux bourgeois des fils et filles du Peuple, « chair à usine, viande à plaisir ».

L'écrivain en recevant «du dehors » ces éléments sociogrammatiques, reçoit et exploite aussi ceux qui composent les traditions propres aux belles-lettres et qui établissent une "phylogenèse » des possibilités thématiques littéraires. Le topos de la prostituée vertueuse (Fleur-de-Marie, Fantine) s'est, depuis le moment romantique, dégradé dans le roman-feuilleton, Chaste et flétri ${ }^{6}$ etc. Le réalisme balzacien a fait, d'autre façon qu'Eugène Sue, de la fille vénale l'allégorie du «mystère » de la société bourgeoise. Baudelaire a fait de la prostituée la figure d'une esthétique anti-naturelle, du charme de l'infamie.

\section{Rôles et emplois}

41 Un «moment » culturel peut être abordé comme la distribution d'une pièce «bien ficelée » à la Scribe, avec ses grands rôles, ses « emplois », ses figurants et ses doublures. Les positions de prestige acquises dans les différents champs et dans l'opinion cultivée en général résultent de la logique hégémonique, des valeurs et thèmes "d'époque ». Chaque rôle incarne une idiosyncrasie thématique et un style particulier. Chaque position est occupée par un seul individu, elle est faite pour lui ; il se l'est appropriée. Il peut avoir d'éventuels challengers cependant, et des alter ego ou des ersatz subalternes (jouant le même rôle à un degré moins raffiné ou moins prestigieux).

Les grandes positions de prestige dans la culture d'une époque, particulièrement celle du Grand Penseur, du Grand Philosophe, titulaire éponyme de la "pensée" de son temps, ne sont pas sous le contrôle du champ philosophique comme tel. On se rappelle la façon dont Antonio Gramsci conçoit la notion de Grand Penseur - il pensait à Benedetto Croce en l'occurrence - et la position-clé qu'il occupe dans la structure idéologique globale. Les "grands esprits» du moment sont littéralement irremplaçables parce qu'ils étaient tellement «de leur temps»! Leur succès, leur influence, leur prestige deviennent en 
partie inexplicables à la génération suivante. La postérité est souvent cruelle pour ces gens dont le prestige a été énorme. Ce prestige est proportionnel à l'oubli qui les ensevelit, après quelques mois de regrets posthumes unanimes. Ils étaient de leur époque, de leur temps. Seul Renan en 1889 fait exception, ce qui suggère que sa pensée, quoique bien « de son époque », était d'une autre qualité.

Le discours social comporte des emplois fixes: pas seulement deux ou trois grands maîtres à penser (rôles qui peuvent être tenus par un personnel posthume), mais d'autres emplois de la scène doxique, jusqu'à des seconds et troisièmes rôles, des utilités et des doublures. On pourrait les nommer, à la façon de la tradition comique avec ses père noble, ingénue, jeune premier, coquette, valet, barbon... Il y aurait, amusons-nous un moment, le Collimateur idéologique (qui met en parallèle les rayons divergents du prisme doxique), l'Énonciateur de paradoxes, le Pervers de service, le Cicerone de l'évasion hors du siècle, l'Arbitre des élégances, le Bourru bienfaisant, les Promoteurs de modes intellectuelles et toutes sortes de propriétaires d'Idées fixes, - rôles sectoriels par essence. Ce qui définit ces «doxosophes» (ainsi que Pierre Bourdieu les a dénommés), c'est leur pouvoir symbolique, un charme à eux qui opère et qui s'impose et qui, avec le " recul », est intégralement dissipé. Ils se définissent par la capacité qu'ils ont eue de se faire applaudir, citer, discuter, prendre en considération par leurs contemporains et de disparaître, pour la plupart, dans le néant du rococo, du passé, de l'illisible une génération plus tard.

Illustration: La scène intellectuelle en 1889. - Ernest Renan est en effet en 1889 le grand intellectuel, - plus que Taine dont l'heure est passée. Un homme s'érige en producteur d'une Weltanschauung éponyme, qui est une variante typée de la vision du monde et de la gnoséologie générale de son temps. Renan est, depuis trente ans alors, le penseur le plus commenté par les savants et par les béotiens. C'est le saint laïc du monde officiel républicain (Renan ne l'est guère, républicain) et de la haute culture. La Troisième république l'a comblé d'honneurs: Académie française, 1878; Administrateur du Collège de France, 1883. Partisan d'une « savantocratie ", élitiste par tempérament, mais honni de l'Église, il est en porte-à-faux, entre les spiritualistes et les matérialistes, entre la droite et la gauche, position que seul un "grand rôle " peut tenir car pour tout autre elle serait trop menacée et instable. Il a influencé de son pyrrhonisme toute une génération. Paul Bourget est, dit-on, son « disciple »; Jules Lemaître est «imbu de renanisme jusqu'aux moelles ». Hors quelques esprits murés dans le ressentiment clérical, Renan suscite une admiration éperdue, fascinée par ses côtés insaisissables: «l'esprit le plus ouvert et le plus pénétrant de son temps ", « le doux philosophe », « l'indulgence universelle », « être d'exception ", « une pure intelligence ", «le maître de ce temps ", " guide de l'esprit » doté d'" un sens divinateur des choses très anciennes ». Grand penseur, mais aussi styliste inimitable ; on vante «le charme de son style chantant et poétique - régal des lettrés ». Il "scandalise" les uns et "ensorcelle» les autres; les Débats concluent que «ce merveilleux esprit est une énigme délicieuse et troublante », lieu commun qui met tout le monde d'accord. Renan offre au discours social, sous ses versions les plus distinguées, une conciliation ironique et ambiguë de toutes les contradictions qui alors «déchirent les esprits ». Il a quitté l'Église, mais respecte la religion tout en niant la divinité du Christ ; annexé par les démocrates, il est favorable à une oligarchie de lettrés et de savants ; progressiste par sa démarche critique, il est bénignement raciste, chauvin et antiplébéien. Ajoutons que pour ceux qui ne suivent pas aisément cette pensée sinueuse, il reste la séduction de l'expression que les mondains jugeaient « délicieuse »: pince-sans- 
rire, plein de sous-entendus ironiques, Renan combine avec bonhomie l'érudition académique et l'esprit boulevardier.

Ce qui caractérise ces "grands rôles", c'est que même ceux qui les haïssent sont contraints d'en parler. L'Univers des Frères Veuillot qui évoque sa « répugnance à parler de M. Renan», en parle sans cesse. Les Études des Jésuites le traite de "répugnant personnage » qui «fait involontairement songer à Judas ». Ce ton grondeur commence à passer de mode. Je ne vois guère que J.-H. Rosny aîné (qui représente la gauche intellectuelle extrême) pour lui reprocher « un nihilisme infécond sacrifiant les virilités nécessaires à un amour excessif de la paix ", ce qui ne l'empêche pas de trouver "profond » le très conservateur Discours de réception de Jules Claretie par Renan. Au fond, même ce mouvement critique atteste, comme les honneurs officiels et les commentaires dévotieux, de l'emprise de Renan sur la France intellectuelle.

Pourtant le renanisme se démodera vite. Henri Bergson, qui publie son premier livre, l'Essai sur les données immédiates en 1889, servira de jeune challenger spiritualiste et contribuera à la mise au rencart intellectuelle de Renan. Renan a rapidement vieilli, diagnostiquera Albert Thibaudet, parce que sa pensée a correspondu de trop près au climat intellectuel de son époque. La pensée de Renan était donc destinée à devenir très vite démodée : Thibaudet ajoutait que le prestige intellectuel immense de Renan a tenu autant à sa force intrinsèque qu'à " son ignorance d'une grande partie de la pensée de son époque ", à un heureux égocentrisme qui le mit "à l'abri des vraies influences de l'époque ».

47 En 1889, un cran plus bas dans l'échelle des distinctions, le grand philosophe pour les philosophiquement faibles, pour le grand public est pourtant Jules Simon, membre de l'Institut, partie prenante à tout ce qui a un prestige officiel en France, Jules Simon est de loin le penseur dont les journaux parlent le plus et dont l'accumulation de capital de légitimité est la plus impressionnante. Sic transit gloria mundi, soupirerons-nous. Le Républicain-spiritualiste-et-pondéré: ce rôle tenu par Jules Simon est tellement nécessaire, il correspond si bien à une attente du discours social que son heureux titulaire est partout: il n'est pas une société savante, une académie, une association philanthropique dont Jules Simon ne soit membre...

L'émergence de rôles doxiques résulte de la division du travail discursif et la question revient à ce "mystère" sociologique de l'harmonie établie entre les dispositions singulières d'un individu et une fonction sociale à remplir. Le tout est de ne point prendre l'effet (l'individu titulaire du rôle) pour la cause. La position qu'occupent ces individus représente un capital social et, plus ou moins consciemment, ils défendent celui-ci des concurrences et contrefaçons. Leur « signature » a en outre une valeur commerciale, pour les rôles les plus publics, grands chroniqueurs, grands journalistes, grands critiques, grands écrivains. Il ne faut pas confondre - bien que l'un engendre les autres - le rôle, défini dans la topologie du discours social, et les positions institutionnelles occupées : Académie, Institut, chronique au Temps ou feuilleton au Journal des Débats. Le rôle est quelque chose de plus impalpable, de plus évanescent que ces positions de grand rapport et c'est, logiquement au moins, lui qui précède et explique les fonctions institutionnelles acquises et occupées.

49 Dans les grands rôles établis à l'avant-scène de toute époque, on peut voir une imposture légitimée : une plus-value de prestige s'attache à leurs moindres propos: Aurélien Scholl est l'Homme-d'esprit-boulevardier: donc tout ce qu'il dit sera spirituel; Renan est le Grand Penseur, il n'a qu'à ouvrir la bouche pour que ce qu'il énonce soit profond, 
important, significatif, digne de gloses et de commentaires. Une autre métaphore vient à l'esprit : celle du Règne. Aurélien Scholl règne sur le «Boulevard »; Francisque Sarcey, sur la Platitude de sens commun et Renan, sur tout l'Empyrée intellectuel. Quand ils sont morts, l'opinion attristée a l'impression que quelque chose meurt avec eux, et d'une certaine manière c'est vrai car ils étaient irremplaçables : Léon Daudet ne remplacera pas Henri Rochefort; il va occuper une position de satiriste de droite dans une topologie renouvelée. Henri Bergson, maitre à penser d'une nouvelle génération se substitue à Renan, mais l'économie interdiscursive entre la philosophie et la doxa globale a complètement changé.

\section{Censures et transgressions, novum et contre- discours} d'une nova au milieu d'une galaxie) d'objets thématiques marqués par les deux formes du "sacer ", de l'intouchable et du sacré: les fétiches et les tabous. Ces intouchables sont connus : ils tentent donc les transgresseurs et les iconoclastes, mais un mana les habite dont témoignent toutes sortes de vibrations rhétoriques à leur abord. La Patrie, l'Armée, la Science sont en 1889 du côté des fétiches ; le Sexe, la Folie, la Perversion sont du côté des tabous. Un grand nombre d'audacieux soulèvent ici le voile d'Isis et s'attirent par leur courage novateur l'approbation des happy few. Ici encore, il faut bien voir qu'un tabou peut en cacher un autre et, aux libertins littéraires notamment, on a surtout envie de dire : encore un effort si vous voulez être vraiment audacieux.

Dire que tel caractère ou telle contrainte discursive, tel interdit ou tabou s'imposent à une époque donnée ne revient pas à exclure que la censure établie n'entre en composition avec de multiples stratégies qui la contestent, l'antagonisent, en grignotent les limites. Ainsi, cas trop connu, il y a en 1889 une censure sur le sexe et sa représentation; je n'en esquisserai pas les caractères en quelques lignes. C'est cette censure même qui permet au libertinage « bien écrit » de Catulle Mendès, à l'apologie boulevardière des cocottes et du Paris des plaisirs dans le Gil-Blas, aux «audaces" sombrement sublimées du roman naturaliste de s'exprimer, d'acquérir du prestige pour certains et de thématiser d'une certaine manière, à la fois dissidente et pourtant bien "de son temps", ses transgressions. Tout ce que les contemporains ont perçu comme nouveau, original, audacieux, inouï (et tout ce que nous percevons comme tel pour notre propre temps et notre culture) risque de ne l'être aucunement, pour la raison même que ce faux inouï ne se perçoit bien comme inouï que parce que les conditions de son marketing sont établies - autrement dit qu'il s'agit d'un inouï immédiatement intelligible contribuant à la récurrence de la doxa dans ses variations.

Il convient donc de chercher à distinguer les rares ruptures réelles qui se rendraient incompatibles avec les dominantes de l'époque et les ruptures ostentatoires et superficielles qui contribuent à l'idéologie même de l'originalité. Le romancier «scandaleux » peut ne faire que confirmer en sous-main les poncifs les plus éculés, le chroniqueur à paradoxes ou le fougueux pamphlétaire peuvent, à leur insu, rester pleinement dans la dépendance des idées qu'ils attaquent, n'en présenter qu'un cas de figure, certes imprévu, mais fondamentalement compatible avec le "système" établi. Ainsi, parce qu'à cette hégémonie, s'attache l'intérêt-de-lecture, un Catulle Mendès et une 
Rachilde en 1889, tout « audacieux » qu'ils étaient, ne sont pas moins devenus « illisibles » aujourd'hui que ne sont les travaux pleins d'autorité du Dr. P. Garnier sur les «aberrations de l'instinct» génésique. Nous voyons bien pourquoi ces écrivains scandaleux ne permettent plus qu'une lecture " archéologique » : perméables aux idées dominantes que leur " perversité » audacieuse se flattait de transgresser, ils ne pouvaient opérer un certain effet «significatif » que dans cette hégémonie propre. On dira qu'ils étaient "de leur temps». Tout paradoxe s'inscrit dans la mouvance d'une doxa. Tout débat ne se développe qu'en appuyant sur une topique commune les conflits des argumentateurs opposés. Dans toute société, la masse des discours - cacophoniques et antagonistes - engendre un dicible global au-delà duquel il n'est possible que par anachronisme de percevoir le « noch-nicht Gesagtes », le pas-encore dit.

Il n'y a pas, il n'y a jamais eu d'émergence tout armée d'un langage neuf dans la tête de quiconque; il n'y a pas, dans l'histoire des discours et des idées, de ruptures (épistémologiques ou autres) franches et irréversibles. Par la nature même des choses, toute rupture est d'abord un glissement de sens mal perceptible, une érosion mal balisée, un balbutiement maladroit. Par maladroit j'entends: qui tâtonne pour se trouver un langage autre, qui ne formule un paradigme hétérodoxe qu'au prix d'un certain aveuglement au potentiel de sa logique nouvelle et qu'en appuyant bien souvent sur des constructions admises par l'époque sans mesurer le conflit interne que suscite la coexistence du légitimé et de l'inouï.

Ce qui peut se repérer occasionnellement dans un état de culture ce sont certains écrits identifiés comme " littéraires » ou non - qui secouent l'entropie des idées reçues ou qui leur tendent un miroir déformant. Ce qui peut se repérer encore ce sont certains textes aussi qui cherchent à donner un langage à ces "choses " que les discours établis ne verbalisent pas - suivant le principe profondément sociologique que ce qui ne se dit pas n'existe pas. Ces textes critiques intéressent évidemment l'historien au plus haut point si le fait discursif doit en effet s'analyser à la fois comme répétition, redondance, compulsion à redire du déjà-dit, comme pré-jugé et mé-connaissance et comme glissements subreptices, comme ironisation, et finalement émergence de logiques autres. L'essentiel, je le répète et ce n'est pas simple, consiste à ne pas confondre ces nouveautés et ruptures authentiques avec ce qu'offre à tout moment en abondance le marché de la Nouveauté culturelle et littéraire, avec ses « retapages ", ses révolutions ostentatoires et ses effets de mode, son «toc » conformiste ou anti-conformiste et son décrochez-moi-ça de l'identité ethnique, sociale et sexuelle.

\section{Discordances cognitives : la notion de non- contemporanéité}

Je termine en posant la question de la « non-contemporanéité » de discours apparemment concomitants. Si l'hégémonie tend à rendre coïntelligibles ou co-acceptables les différents discours légitimes, il doit pouvoir être possible cependant de repérer, sur les " marges » peut-être, des pratiques discursives qui tout en étant chronologiquement contemporaines, sont non seulement antagonistes, mais plus encore " incompossibles ", les produits de démarches cognitives, de "gnoséologies » incompatibles qui manifestent dans une coexistence trompeuse la contemporanéité de discours non-contemporains. La notion d'Ungleichzeitigkeit à laquelle je me rapporte vient d'Ernst Bloch (1934): elle s'appliquait à ce qu'il percevait d'« anachronique », de pulsions pré-capitalistes dans les 
idéologies et les «attitudes mentales» des Nazis : «Tous ne sont pas présents dans le même temps présent, écrivait-il. Ils n'y sont qu'extérieurement ... Ils portent avec eux un passé qui s'immisce ... Des temps plus anciens que ceux d'aujourd'hui continuent à vivre dans des couches plus anciennes ».

Le discours clérical catholique vers la fin du $19^{e}$ siècle, enfermé dans la logique antimoderniste du Syllabus errorum de Pie IX, considérant comme peccamineuses la presse, la littérature, la science laïques, est un excellent exemple de l'Ungleichzeitigkeit. Le contre-discours catholique, voulu bigot, réactionnaire, antirationnel, se faisant gloire d'une arriération mentale méticuleusement entretenue n'est pas non contemporain au sens où il serait une survivance (comme le sont certaines "mentalités » paysannes); il représente un "archaïsme de combat » dont la vision apocalyptique du monde moderne n'est pas sans interférer d'ailleurs avec les angoisses de la déstabilisation symbolique qui s'expriment un peu partout.

J'évoquerai pour finir avec une image suggestive la rencontre de Don Quichotte et des Marchands. Antonio Gómez-Moriana (1988) en a fait une subtile analyse. Elle fait voir la discordance cognitive entre humains qui se rencontrent sur une même route comme l'objet par excellence de l'ironie romanesque à la naissance même du genre. Don Quichotte met en demeure des marchands qui ont croisé sa route de confesser que Dulcinea del Toboso est la plus belle dame de l'univers. Les marchands, interloqués par ce bravache, mais qui ne veulent pas faire d'histoire et appartiennent à une "mentalité " que nous appellerons moderne, mercantile et pratique, font remarquer au noble chevalier que s'il exhibait un camée ou un portrait de la gente dame, ils pourraient juger sur pièce. À quoi, l'homme de la Manche, indigné, réplique avec feu que s'il leur montrait un portrait de Dulcinée, ils n'auraient évidemment aucun mérite à admettre le fait et qu'il leur convient de reconnaître les charmes de la dame sur sa parole. Une logique de l'honneur féodal de l'archaïque Don Quichotte s'oppose, dans ce dialogue de sourds et cette " coupure » mentalitaire, à une logique « expérimentale » qui en est l'opposé. Cet épisode comique est présenté par Cervantès, à l'orée de la modernité, comme la rencontre de deux univers mentaux non-contemporains, ungleichzeitig, qui demeureront absurdes et choquants l'un pour l'autre?

\section{BIBLIOGRAPHIE}

Angenot Marc, Le Cru et le faisandé : sexe, discours social et littérature à la Belle Époque, Bruxelles, Labor, 1986.

Angenot Marc, Mil huit cent quatre-vingt-neuf : un état du discours social, Montréal, Longueuil, Éditions du Préambule, 1989a.

Angenot Marc, Ce que l'on dit des Juifs en 1889. Antisémitisme et discours social, Paris, SaintDenis, Presses de l'Université de Vincennes, $1989 \mathrm{~b}$.

Angenot Marc, Le Centenaire de la Révolution, Paris, La Documentation française, 1989c.

Angenot Marc, Topographie du socialisme français, 1889-1890, Montréal, Discours social, 1990. 
Marc Angenot, Dialogues de sourds, Paris, Mille et une nuits, 2008.

Marc Angenot et Régine Robin, « L'Inscription du discours social dans le texte littéraire », Sociocriticism, vol. I, $\mathrm{n}^{\circ}$ 1, 1985. pp. 53-82.

Barthes Roland, Mythologies, Paris, Seuil, 1957.

Bloch Ernst, Erbschaft dieser Zeit, Berlin, 1934.

Debray Régis, L'Emprise, Le Débat-Gallimard, 2000.

Duchet Claude, Le réel et le texte, Paris, Colin, 1974.

Duchet Claude, « La mise en texte du social », Balzac et la Peau de chagrin, Paris, SEDES, 1979a, pp. 79-92.

Duchet Claude, « Positions et perspectives », Sociocritique, Paris, Nathan, 1979b, pp. 3-9.

Duchet Claude, «Idéologie de la mise en texte », La Pensée, n 215, 1980, pp. 95-108.

Foucault Michel, Les Mots et les choses, Paris, Gallimard, 1966.

Foucault Michel, Archéologie du savoir, Paris, Gallimard, 1969.

Ginzburg Carlo, Il formaggio e i vermi, Einaudi, 1976.

Gómez-Moriana Antonio, « Discourse Pragmatics and Reciprocity of Perspective: The Promise of Juan Haldudo ("Don Quijote", I, 4) and of Don Juan», Sociocriticism, 1988, 4,1 (7), 87-109. [En espagnol dans la Nueva Revista de filología hispánica, XXXVI-2, 1988].

Miller Perry, The New England Mind : the 17th Century, Harvard UP, 1954, republication en 1982.

Tillyard Eustace, The Elizabethan World Picture, London, Chatto \& Windus, 1943.

\section{NOTES}

1. Weltanschauung apparaît chez Kant. Le mot a connu une complexe histoire philosophicosémantique en allemand avec Schlegel, Hegel, Søren Kierkegaard - en allemand dans le texte danois -, Dilthey, Nietzsche, Husserl, Jaspers - et un succès académique dont témoigne la haute fréquence du mot mis à «toutes les sauces » dans les titres de thèses de lettres et de philosophie en Allemagne depuis le début du $20^{\text {e }}$ siècle jusqu'à 1945 - date à laquelle la nazification du syntagme en assure la déconsidération. Le mot qui n'apparaît, en emprunt de l'allemand ou transposé en français, que vers 1930 n'a jamais eu dans notre langue un sens bien établi ni une fréquence semblable. "Vision du monde» est parfois utilisé par des critiques toutefois pour désigner la philosophie "spontanée », la cosmologie, l'anthropologie, la politique et la morale implicites des artistes, des écrivains, des poètes, des romanciers, des dramaturges. On parlera de la « vision du monde » de Stendhal, de Balzac, de Flaubert, de celle des surréalistes etc. : à savoir les réponses données par de grands esprits qui ne furent pas des philosophes "attitrés ", sous forme lyrique ou romanesque, aux questions fondamentales, Qui sommes-nous, d'où venonsnous, où allons-nous? De même se fait-on comprendre en évoquant la "vision du monde » de Shakespeare, de Goethe, de Darwin, de Renan, de Taine etc., plutôt que de parler abusivement de leurs «philosophies ».

2. Le premier de ses livres étant Le degré zéro de l'écriture.

3. Je songe à Quentin Skinner, notoire historien des fondements de la pensée politique moderne, spécialiste de la Renaissance et de l'Âge classique. Il est le chef de file de ce qu'on désigne comme «l'École de Cambridge » à laquelle on rattache d'autres grands chercheurs, John Greville Agard Pocock et John Dunn. 
4. C'est-à-dire celle de Wundt bien avant Freud.

5. Sur ma propre définition du concept, voir Angenot et Robin (1985).

6. Roman de Pierre Decourcelle.

7. J'ai alors proposé la description contrastée de quatre grandes «logiques» concurrentes qui s'affrontent au cours de la modernité, quatre grands types de singularités rhétoriques, relevés au cours de mes études de la pensée politique et sociale des deux derniers siècles. Angenot (2008).

\section{RÉSUMÉS}

L'auteur aborde l'approche synchronique en histoire des idées et développe la théorie du discours social qu'il a exposée dans nombre de ses livres en tant qu'analyse en totalité en coupe synchronique de ce qui s'écrit et se diffuse dans un état de société. Il définit dans le cadre de cette problématique les notions fondamentales d'hégémonie discursive, de topographie, d'interdiscursivité et de sociogramme notamment.

In the general framework of a synchronic approach of intellectual history, the author defines the basic concepts of Social Discourse Theory seen as the method of a all-encompassing analysis of whatever is being written, printed and disseminated in a given state of society. He aims at defining a number of fundamental notions such as Discursive hegemony, Topography, Interdiscursiveness and Sociogramme.

\section{INDEX}

Mots-clés : Esprit du temps (Zeitgeist), Discours social, Synchronie vs diachronie, Hégémonie, Marché discursif, Topographie, Division du travail, Interdiscursivité, Migrations, Sociogramme, Emploi [théâtre], Novum, Contre-discours, Non-contemporanéité (Ungleichzeitigkeit)

Keywords : Spirit of the Age (Zeitgeist), Social discourse, Synchrony and diachrony, Hegemony, Discursive market, Topography, Division of labor, Inter- Discursiveness, Migrations, Stock character, Novum, Counter-discourse, Ungleichzeitigkeit

\section{AUTEUR}

\section{MARC ANGENOT}

Université McGill à Montréal 